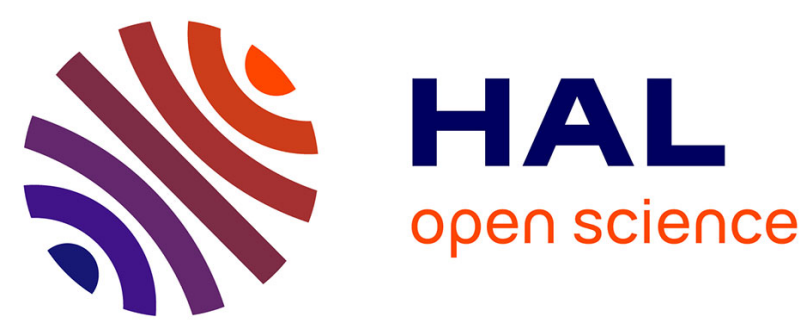

\title{
Lamella Division in a Foam Flowing through a Two-Dimensional Porous Medium: A Model Fragmentation Process
}

Baudouin Géraud, Yves Méheust, Isabelle Cantat, Benjamin Dollet

\section{- To cite this version:}

Baudouin Géraud, Yves Méheust, Isabelle Cantat, Benjamin Dollet. Lamella Division in a Foam Flowing through a Two-Dimensional Porous Medium: A Model Fragmentation Process. Physical Review Letters, 2017, 118 (9), pp.098003. 10.1103/PhysRevLett.118.098003 • hal-01483090

\section{HAL Id: hal-01483090 https://hal.science/hal-01483090}

Submitted on 4 Mar 2017

HAL is a multi-disciplinary open access archive for the deposit and dissemination of scientific research documents, whether they are published or not. The documents may come from teaching and research institutions in France or abroad, or from public or private research centers.
L'archive ouverte pluridisciplinaire HAL, est destinée au dépôt et à la diffusion de documents scientifiques de niveau recherche, publiés ou non, émanant des établissements d'enseignement et de recherche français ou étrangers, des laboratoires publics ou privés. 


\title{
Lamella division in a foam flowing through a two-dimensional porous medium: A model fragmentation process
}

\author{
Baudouin Géraud, ${ }^{1}$ Yves Méheust, ${ }^{2}$ Isabelle Cantat, ${ }^{1}$ and Benjamin Dollet ${ }^{1}$ \\ ${ }^{1}$ Institut de Physique de Rennes, UMR 6251 CNRS and Université Rennes 1, 35042 Rennes Cedex, France \\ ${ }^{2}$ Géosciences Rennes, UMR 6118 CNRS and Université Rennes 1, 35042 Rennes Cedex, France
}

(Dated: February 9, 2017)

\begin{abstract}
We flow a $2 \mathrm{D}$ foam through a model 2D porous medium and study experimentally and numerically how the bubble size distribution evolves along the medium. The dominant mechanism of bubble creation is a fragmentation process occurring when bubbles pinched against obstacles are split in two smaller bubbles. We infer the statistics of these individual and local fragmentation events from the experimental data and propose a fragmentation equation to relate that statistics to the evolution of the global size distribution. The predicted evolution shows very good agreement with direct experimental measurements of the bubble size distribution.
\end{abstract}

PACS numbers:

Among the various applications of liquid foams [1], foam flows in porous media are particularly important in various subsurface applications. They were first used in enhanced oil recovery more than 50 years ago [2] due to their viscosity ratio with oil less unfavorable than that of water, and later, for the same reason, to remediate aquifers contaminated with nonaqueous phase liquids [3]. More recently they have been utilized in the remediation of polluted soils [4], because of their low cost, much smaller sensitivity to gravity than aqueous liquids, and of the biodegradation enhancement that they offer [5]. Common to all these applications is the good sweep efficiency of foams, which has been related to their complex mobility in porous media [6, 7], characterized by their shear thinning behavior which holds even under confinement in narrow pores [8-10], and by processes of destruction and creation of films (the so-called leave-behind, lamella division and snap-off mechanisms [11-13]), which impact the bubble size distribution. The foam mobility in porous media is known to strongly depend on this bubble size distribution [6]. A good understanding of its evolution is thus crucial for all situations in which the pressure drop and foam velocity need to be controlled. However, it is difficult to characterize foams in core flooding units or column experiments in situ, which has led us to study foam flows in transparent porous media. In this context, we have investigated the local rheology of a two-dimensional (2D) foam flowing in a transparent 2D porous medium (shown in Fig. 1), and characterized its phenomenology as a function of various control parameters [14]. We observed an irreversible evolution of the bubble size distribution, mostly dependent on the water content and initial mean bubble area. We have identified lamella division as the predominant mechanism of bubble size evolution for that medium. It consists in the splitting of a bubble (henceforth called mother bubble) in two fragments (henceforth called daughter bubbles) when its upstream film spans two obstacles and impinges on a third one (the "fragmenting obstacle", see Fig. 1),
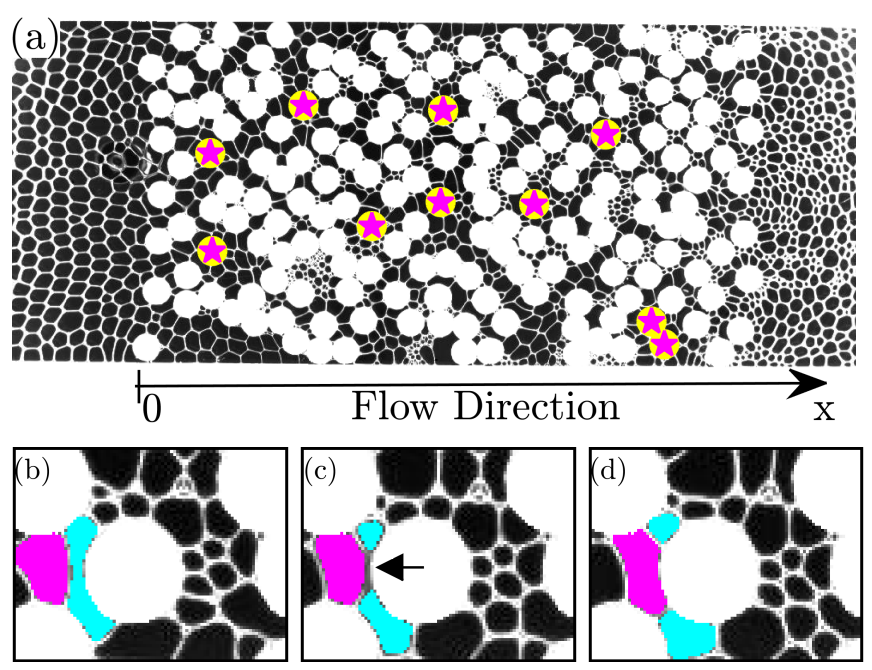

FIG. 1: (a) Snapshot of the whole foam flow cell. (b, c, d) Close-ups on a bubble fragmentation. The time interval between frames (b), (c) and (d) is $50 \mathrm{~ms}$. The obstacle diameter is $7.5 \mathrm{~mm}$. In (c), an arrow shows the location where the splitting lamella touches the obstacle, triggering the fragmentation event. The mother bubble (b) and the two fragments (c, d) are colored in cyan.

In this Letter, we propose the first model of the evolution of the bubble size distribution as a foam flows along a porous medium in which bubbles are subjected to lamella division. We relate this evolution to the individual fragmentation events. Obtaining from their statistics a size-dependent fragmentation rate and a distribution of fragment sizes, we propose a model based on a fragmentation equation [15], which fits our data quantitatively. Notably, we show that such a model allows to rescale different experiments, thereby offering a universal prediction of the size evolution based on a small number of parameters. Our study thus pertains to the general field of fragmentation processes, which are ubiquitous in chemical engineering [16], polymer degradation [17], cel- 
lular division [18], grinding or liquid atomization [19].

We used a Hele-Shaw cell of gap $2 \mathrm{~mm}$, width $9.6 \mathrm{~cm}$ and length $27 \mathrm{~cm}$. The porous medium spans a length of $17.8 \mathrm{~cm}$ in the middle of this channel. It consists of an assembly of monodisperse plastic disks of thickness $2 \mathrm{~mm}$ and diameter $7.5 \mathrm{~mm}$, which were inserted and glued manually. The positions of these obstacles were defined from a computer-generated geometry obtained by perturbing a diagonal regular mesh with a random displacement drawn from a Gaussian distribution. The average pore size, defined as the distance between two neighboring disks (in the sense of their Delaunay triangulation), is $4 \mathrm{~mm}$. The porosity of the porous medium thus created is 0.5 . The cell is connected upstream with a vertical chamber filled with a solution of sodium dodecyl sulfate of concentration $10 \mathrm{~g} / \mathrm{L}$ in ultrapure water. The foam is created by bubbling nitrogen in the solution through needles; it is then pushed into the cell at a prescribed flux. Bubbles are large enough to form a monolayer, i.e. a two-dimensional foam, when confined within the cell gap. The foam is initially fairly monodisperse, the bubble area distribution being peaked around a characteristic value $a_{0}$. Both the fluxes of the gas, $Q_{g}$, and of the solution, $Q_{\ell}$, are controlled and constant, so that our experiments correspond to steady flows of mean velocity $V_{0}$. The flux ratio, $\alpha=Q_{\ell} / Q_{g}$, controls the liquid fraction [14].

If the bubble size of the foam entering the channel is larger than the pore size, snapshots of the experiments (Fig. 1) show clearly that the bubbles at the outlet are on average smaller than those at the inlet. To quantify this observation, we measure the evolution of the bubble area distribution along the porous medium as follows. We measured bubble sizes after skeletonization of the raw images. We meshed the porous medium into 16 slices, each of streamwise length $\Delta x=1.1 \mathrm{~cm}$ and spanning the full width of the channel. On each slice and each image, we recorded the area of the bubbles whose center lies inside the slice. This gives the density of bubble numbers per unit length and bubble area $n(a, x)$, where $x$ is the longitudinal coordinate of the system, and $a$ the bubble area, $n(x, a) \Delta x \Delta a$ is the number of bubbles of area between $a$ and $a+\Delta a$ comprised in the longitudinal range $[x, x+\Delta x]$. The origin $x=0$ is taken at the inlet of the porous medium. Data are accumulated over time (2000 images per run at $20 \mathrm{fps}$ ) to increase the statistics, thanks to the steady character of the mean flow. The resulting evolution of the bubble area distribution is plotted in Fig. 2 for two experiments A and B of different initial bubble area $a_{0}$ and liquid fraction, as a function of the reduced area $\bar{a}=a / a_{0}$. As $x$ increases, the initial peak decreases, and a skewed peak of smaller bubbles grows. Accordingly, the average bubble size $\langle a\rangle$ decreases, as shown in Fig. 3b for experiment A.

The elementary mechanism responsible for the decrease of bubble sizes is readily found by visual inspection of the movies: it is the aforementioned process of lamella division (Fig. 1). It is reminiscent of the fragmentation on an obstacle of drops or bubbles confined in microchannels $[20,21]$. No fragmentation in a higher number of fragments was observed, nor other fragmentation processes; in particular, the snap-off of films $[12,13]$ does not occur in our experiments because the pore size is much larger than the soap films coating them. On the other hand, we observed no lamella destruction events [22]. Hence, our experimental conditions enabled us to focus on the influence of the sole bubble splitting by lamellar division on the bubble size distribution.

To model this fragmentation process, we assume that for a given streamwise distance $\Delta x$, the probability of a bubble of a given size $a$ to break between $x$ and $x+\Delta x$ is of the form $F(a) \Delta x$ and does not depend on $x$. Indeed, due to the randomness of the porous geometry, it is reasonable to assume a uniform distribution of fragmentation sites. As a mother bubble splits, it yields fragments of sizes $b$ and $a-b$ according to a distribution $G(b \mid a)$, defined for $b \in] 0, a[$. Since only two fragments are produced for each event, and since fragmentation conserves the area, the following three constraints apply: $\int_{0}^{a} G(b \mid a) \mathrm{d} b=2, G(b \mid a)=G(a-b \mid a)$, and $\int_{0}^{a} b G(b \mid a) \mathrm{d} b=a$. Hence, the evolution of the bubble area distribution is ruled by a fragmentation equation of the form: $\partial n(a, x) / \partial x=-F(a) n(a, x)+$ $\int_{a}^{\infty} G(a \mid b) F(b) n(b, x) \mathrm{d} b$. The first term in the right-hand side quantifies the loss of bubbles of size $a$ by fragmentation, while the second one quantifies its gain as bigger bubbles split, resulting in fragments of size $a$. This equation is well documented in contexts such as crushing, grinding or polymer degradation [15, 17, 23]. Note that if the integral in the right-hand side of the fragmentation equation above vanishes, the equation reduces to a simple differential equation of exponential solution. Since there are very few bubbles of size larger than the initial mean bubble size $a_{0}$, this assumption is actually valid for the number density of bubbles of size $a_{0}$, hence we expect $n\left(a_{0}, x\right) \propto \mathrm{e}^{-F\left(a_{0}\right) x}$. Plotting $n\left(a_{0}, x\right)$ as a function of $x$ indeed yields an exponential decrease (Fig. 3a), which provides $F\left(a_{0}\right)=14.5 \pm 2.1 \mathrm{~m}^{-1}$. We then define the dimensionless variables of the system: $\bar{a}=a / a_{0}$, $\bar{x}=x F\left(a_{0}\right), f(a)=F(a) / F\left(a_{0}\right)$ and $g(b \mid a)=a_{0} G(b \mid a)$. The number density of bubbles, $n(\bar{a}, \bar{x})$, then follows a fragmentation equation:

$$
\frac{\partial n}{\partial \bar{x}}(\bar{a}, \bar{x})=-f(\bar{a}) n(\bar{a}, \bar{x})+\int_{\bar{a}}^{\infty} g(\bar{a} \mid \bar{b}) f(\bar{b}) n(\bar{b}, \bar{x}) \mathrm{d} \bar{b} .
$$

At small enough velocities, the fragmentation events are controlled by the porous geometry and bubble shape, and can be reproduced numerically [24]. However, the inferred statistics for $f$ and $g$ in random media is still unknown, and needs to be measured. To achieve this goal, we used a statistical sample of splitting events in experiment A. We identified the 10 fragmenting obstacles 

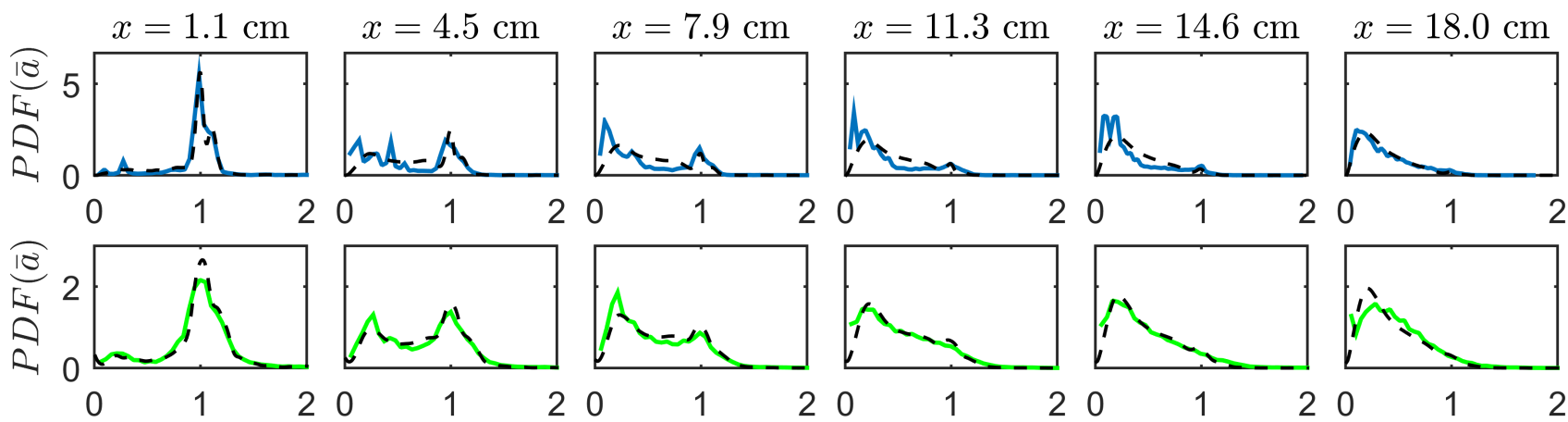

FIG. 2: Evolution of the bubble size distributions at several positions along the porous medium. The blue and green continuous lines denote the experimental measurements. The dashed lines indicate the simulations predictions, based on the sole knowledge of the functions $f, g$ and of the initial size distribution. Top (blue curves, experiment A): wet foam, flow parameters: $Q_{g}=$ $100 \mathrm{~mL} / \mathrm{min}, Q_{l}=10.7 \mathrm{~mL} / \mathrm{min}, a_{0}=19.2 \mathrm{~mm}^{2}$. Bottom (green curves, experiment B): dry foam, flow parameters: $Q_{g}=$ $100 \mathrm{~mL} / \mathrm{min}, Q_{l}=1.5 \mathrm{~mL} / \mathrm{min}, a_{0}=12.5 \mathrm{~mm}^{2}$.
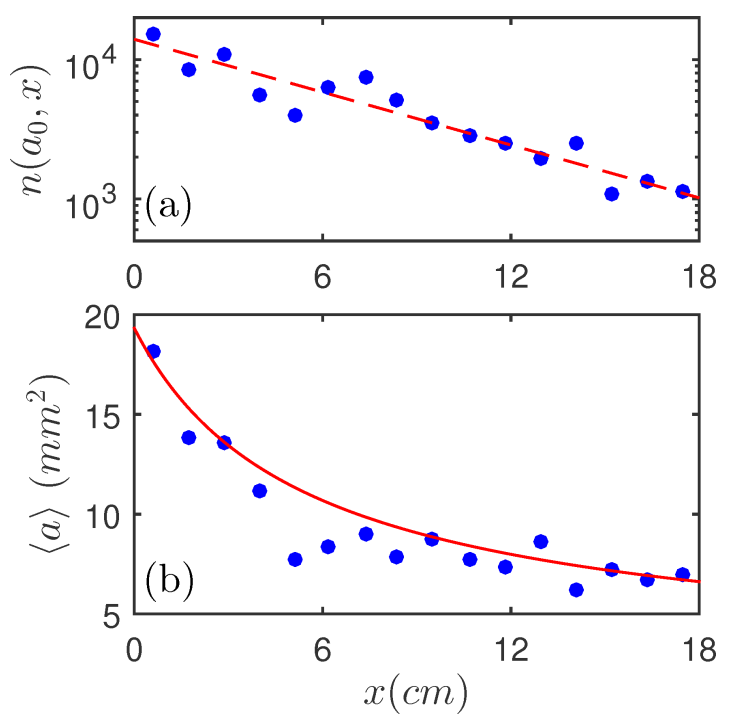

FIG. 3: For experiment A: (a) evolution of the number of bubbles of size $a_{0}$ along the mean flow direction. The blue dots are measurements across the channel and the dashed red line is the exponential fit $n\left(a_{0}\right) \propto \mathrm{e}^{-F\left(a_{0}\right) x}$, with best fitting parameter $F\left(a_{0}\right)=14.5 \pm 2.1 \mathrm{~m}^{-1}$. (b) Evolution of the average bubble size. Blue dots are experimental measurements, and the continuous red line is the simulation prediction.

(marked by a star in Fig. 1) at which most fragmentation events occurred, and detected all fragmentation events at these sites by automatic image treatment. For each site and each fragmentation, we measure the size of the mother bubble and the sizes of the two daughter bubbles, using a procedure described in the Supplementary Materials, which includes Refs. [25-27]. We thus recorded a list of 563 mother bubble areas, $N_{\text {frag }}(a)$, with a minimum $a_{\min }$ and a maximum $a_{\max }$.

To deduce the fragmentation rate from these records, we need to account for the local bubble size distribution. We thus record the area of all bubbles touching the se- lected fragmenting obstacles on each image, which gives a second list of areas, $N(a)$, of the bubbles surrounding these obstacles. Bubbles can thus be counted several times, but this does not bias the distribution since the residence time in a given site was observed to be roughly constant for all bubbles. To estimate the fragmentation rate, we mesh the interval $\left[a_{\min }, a_{\max }\right]$ into ten bins of equal size, and for each bin we compute the ratio $N_{\text {frag }}(a) / N(a)$, which is proportional to the fragmentation rate. To keep a reasonable statistical significance, we discard the bins with less than ten mother bubbles; they concern most sizes above the initial peak distribution, where both $N_{\text {frag }}(a)$ and $N(a)$ are small. The six remaining data points for $N_{\text {frag }}(a) / N(a)$ are well fitted by a power law, of exponent $1.9 \pm 0.3$ (Fig. 4). At this stage, the prefactor is arbitrary, since it depends on the frame rate and on the number of chosen fragmentation sites: to correct this, we rescale the fitting curve of Fig. 4 by the value for $F\left(a_{0}\right)$, known from the fitting procedure of Fig. 3a. This process finally yields:

$$
f(\bar{a})=F(a) / F\left(a_{0}\right)=\left(a / a_{0}\right)^{1.9} .
$$

Concerning the fragmentation kernel, we assume for simplicity that it does not depend on $a$ and $b$ independently, but on the sole relative size $b / a$ of the fragments. This amounts to assuming that $g$ has a self-similar form $g(b \mid a)=g_{0}(b / a) / a$. Our procedure yields the experimental kernel in Fig. 4b. As expected, it is symmetric with respect to $a / b=1 / 2$. Interestingly, it is bimodal, with peaks for $a / b=0.3$ and 0.7 and a shallow minimum at 0.5 , and it decreases strongly towards 0 and 1 , meaning that a fragmentation in two daughter bubbles of very different sizes is an unlikely event.

We then relate this local characterization to the evolution of the bubble size distribution along the channel for experiment A. We plug the experimentally determined form (2) of the frequency rate $f$, and a polynomial fit of the fragmentation kernel $g$ (Fig. 4), in the fragmentation 

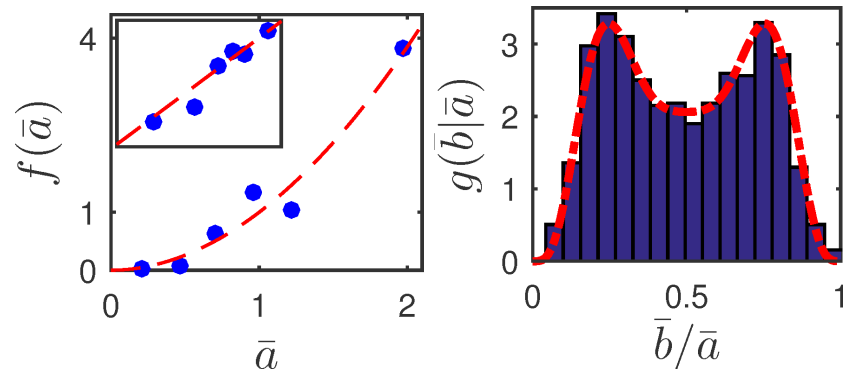

FIG. 4: Fragmentation functions obtained for experiment A. Left panel: $f(\bar{a})$ (inset: corresponding log-log plot), and right panel: $g(\bar{b} / \bar{a})$. The lines are respectively a power law fit for $f$, yielding as a best fitting parameter an exponent $1.9 \pm 0.3$, and an empirical polynomial fit of degree 12 for $g$, symmetric with respect to $\bar{b} / \bar{a}=1 / 2$.

equation (1), and simulate this equation with an explicit Euler discretization detailed in the Supplementary Material. The results of the simulation are overlaid with the experimental measurements in Fig. 2 (top). They show good agreement: the erosion of the initial peak is well captured as expected (since $f$ has been calibrated from that erosion), but more importantly the bubble size distribution at the medium outlet is well predicted as a skewed population of bubbles smaller than the initial bubble size, $a_{0}$. The agreement is better towards the outlet. As a likely reason, the foam exiting the channel has undergone all possible fragmentation events, which makes the validity of the mean-field approximation underlying the fragmentation equation more potent than close to the inlet, where the first deviation from the initial distribution is dictated by a few specific events. Note also that the simulated distribution features an initial rise from 0 at the smallest bubble sizes, which is not always seen in the experimental data. This is due to possible artifacts in the skeletonization of very small bubbles during image processing.

To assess the generality of the functions $f$ and $g$, we keep their values obtained from experiment $A$, and now perform the comparison on experiment B. We only measure the decay of the initial bubble area on this new experiment, which provides $F\left(a_{0}\right)=8.2 \pm 0.7 \mathrm{~m}^{-1}$ for experiment B. Here again, the agreement between the experiments and the simulations is excellent (Fig. 2, bottom). More generally, our model based on (1), if combined with the assumption that the functions $f$ and $g$ are only controlled by the medium's geometry, suggests that for a given initial distribution, the evolution of the size distribution should be similar when the bubble area is rescaled by the mean bubble area $a_{0}$ of the foam entering the porous medium, and when $x$ is rescaled by the fragmentation rate of bubbles of size $a_{0}$. To test this, we plot $n(\bar{a}, \bar{x})$ at $\bar{x}=1$ for 8 different experiments in Fig. 5 with different initial mean bubble areas $a_{0}$, mean velocities $V_{0}$, flux ratios $\alpha=Q_{\ell} / Q_{g}$, and slightly different realiza- tions of the geometrical disorder of the porous medium, but similar initial bubble size distributions: they are all symmetrical (to a good approximation) around the mean bubble size and of standard deviation $\delta \bar{a}=0.10 \pm 0.02$. It shows that within some scatter on the measurements of the distributions, all rescaled distributions collapse on a single master curve. Hence, the assumption that the functions $f$ and $g$ do not depend on the initial mean bubble size, mean flow velocity, and water content of the foam, is reasonable. Likewise, the power law exponent of Eq. (2) also seems to hold for all experiments. In other words, $f$ and $g$ appear to be universal features of the foam fragmentation in the sense that they depend primarily on the medium geometry, irrespective of the foam properties, which set $F\left(a_{0}\right)$. The impact of a stronger polydispersity of the initial bubble size distribution on the fragmentation process has been studied numerically (see the Supp. Mat.). The evolution at small $\bar{x}$ was found to depend on the initial polydispersity, but not the asymptotic size distribution at large enough $\bar{x}$.

The sole dependence of $f$ and $g$ on the medium geometry can only be asserted on the investigated range of bubbles sizes, though; in particular, a lower cutoff is expected in the power law form of the fragmentation rate, since bubbles that are sufficiently small with respect to the obstacle size are expected to slide around the obstacles rather than experience fragmentation. On the other hand, the lamella division process is purely local: it involves one lamella and three obstacles, regardless of the size of the mother bubble. In particular, we expect $f$ to remain scale invariant, as (2), for larger bubbles, until they span the full channel length. We observed that long bubbles align along single preferential paths, which sets an upper bound of bubble areas for the validity of our model, of the order of the product of the average pore size by the medium length, i.e. $7 \times 10^{2} \mathrm{~mm}^{2}$ in our case. In addition, since the porous geometries used for the different experimental runs are slightly different realizations of a porous medium defined by its porosity and the statistical description of its disorder and connectivity, we can even argue that $f$ and $g$ are universal for a given such statistical description of the geometry.

The independence of $f$ and $g$ on the mean flow velocity is valid for the experimentally explored velocities, but may falter at mean flow velocities sufficiently large for the flow to depart from the quasistatic regime, for which lamella shapes are controlled by minimum surface laws, so that the viscoelastic properties of the bubble surfaces do not impact the displacement of lamellas. For the solution used in our experiments, the quasistatic regime holds up to velocities up to $40 \mathrm{~cm} / \mathrm{s}$ [28]. Since the maximal velocity within the porous medium reaches $13 V_{0}$ [14], it thus holds up to $V_{0} \approx 3 \mathrm{~cm} / \mathrm{s}$.

In conclusion, we have characterized in details, at the local and global scale, the process of fragmentation of a foam in a porous medium in which lamella division is the 


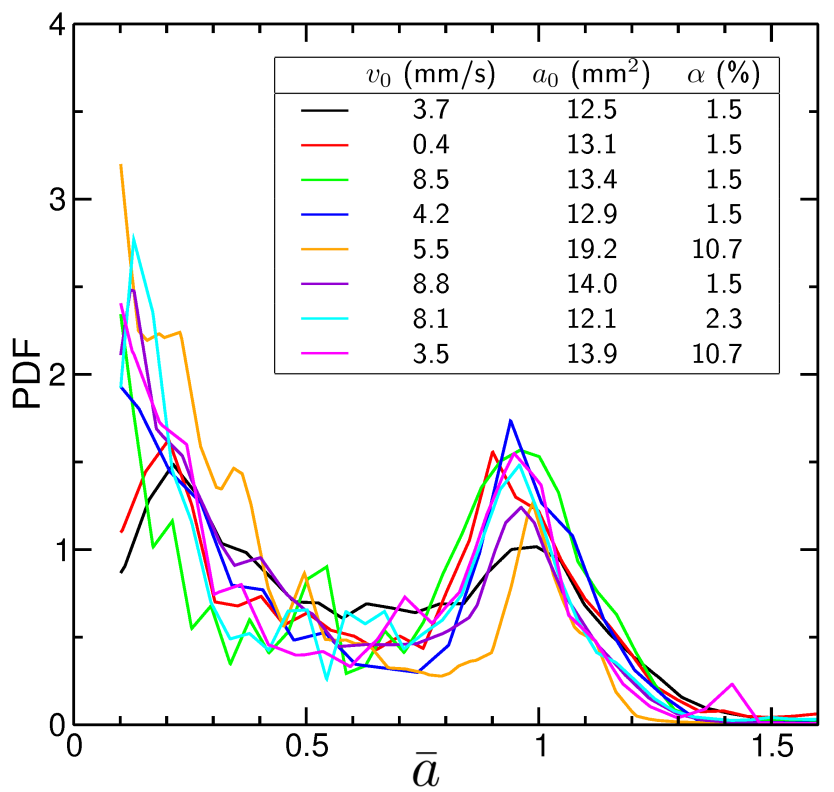

FIG. 5: Plot of bubble size distributions for various experiments, rescaled by the mean area $a_{0}$ of each initial distribution, at a given rescaled location $\bar{x}=1$ within the porous media. The parameters of the experiments are indicated in the legend.

dominant mechanism of change in the number of flowing lamellas. The experimentally characterized evolution is well captured by a predictive fragmentation model based on statistical functions (fragmentation rate and fragmentation kernel) that are inferred from the experimental data. This study opens several interesting questions: what are the mechanisms explaining the power law behavior of the fragmentation rate and the detailed form of the fragmentation kernel, and how are these impacted by the statistical properties of the porous medium. This could lead to a rational control of the bubble size distribution, and thus of the foam mobility, reached at a given travel length in a porous medium where bubble fragmentation by lamella division is the main mechanism of lamella creation. Another perspective would be to study foam fragmentation dominated by snap-off mechanisms, which are expected to be promoted in conditions opposite to ours: higher liquid fraction, smaller pores (e.g. in the micrometric range) forming long, slender interconnected channels $[11,29]$.

[1] P. Stevenson, Foam Engineering: Fundamentals and Applications, Wiley (2012).
[2] S. H. Talebian, R. Masoudi, I. M. Tan, P. L. J. Zitha, J. Pet. Sci. Technol. 120, 202 (2014).

[3] S. Fiorenza, C. A. Miller, C. L. Oubre, C. H. Ward, NAPL Removal: Surfactants, Foams, and Microemulsions, Lewis Publishers (2016).

[4] S. W. Jeong, M. Y. Corapcioglu, S. E. Roosevelt, Environ. Sci. Technol. 34, 3456 (2000).

[5] R. K. Rothmel, R. W. Peters, E. S. Martin, M. F. DeFlaun, Environ. Sci. Technol. 32, 1667 (1998).

[6] A. R. Kovscek, H. J. Bertin, Transp. Porous Media 52, 17 (2003).

[7] A. R. Kovscek, H. J. Bertin, Transp. Porous Media 52, 37 (2003).

[8] G. Hirasaki, J. B. Lawson, Soc. Pet. Eng. J. 25, 176 (1985).

[9] A. Falls, J. Musters, J. Ratulowski, SPE Reservoir Eng. 4, 155 (1989).

[10] I. Cantat, Phys. Fluids 25, 031303 (2013).

[11] A. R. Kovscek, C. J. Radke, in Foams: Fundamentals and Applications in the Petroleum Industry, edited by L. L. Schramm, ACS Publ. (1994).

[12] K. Ma, R. Liontas, C. A. Conn, G. J. Hirasaki, S. L. Biswal, Soft Matter 8, 10669 (2012).

[13] R. Liontas, K. Ma, G. J. Hirasaki, S. L. Biswal, Soft Matter 9, 10971 (2013).

[14] B. Géraud, S. A. Jones, I. Cantat, B. Dollet, Y. Méheust, Water Resour. Res. 52, 773 (2016).

[15] Z. Cheng, S. Redner, Phys. Rev. Lett. 60, 2450 (1988).

[16] J. M. Coulson, J. F. Richardson, Chemical Engineering, Pergamon (1968).

[17] R. M. Ziff, E. D. McGrady, Macromolecules 19, 2513 (1986).

[18] B. Perthame, L. Ryzhik, J. Differential Equations 210, 155 (2005).

[19] E. Villermaux, Annu. Rev. Fluid Mech. 39, 419-446 (2007).

[20] S. Protière, M. Z. Bazant, D. A. Weitz, H. A. Stone, EPL 92, 54002 (2010).

[21] L. Salkin, L. Courbin, P. Panizza, Phys. Rev. E 86, 036317 (2012).

[22] Z. Khatib, G. Hirasaki, A. Falls, SPE Reservoir Eng. 3, 919 (1988).

[23] K. J. Reid, Chem. Eng. Sci. 20, 953 (1965).

[24] S. J. Cox, Colloids Surf. A 473, 104 (2015).

[25] B. Dollet, S. A. Jones, Y. Méheust, I. Cantat, Phys. Rev. E 90, 023006 (2014).

[26] B. Dollet, A. Scagliarini, M. Sbragaglia, J. Fluid Mech. 766, 556 (2015).

[27] B. Dollet, C. Bocher, Eur. Phys. J. E 38, 123 (2015).

[28] B. Dollet, I. Cantat, J. Fluid Mech. 652, 529 (2010).

[29] A. R. Kovscek, G. Q. Tang, C. J. Radke, Colloids Surf. A 302, 251 (2007). 